\title{
La atención a las personas con discapacidad en las universidades mexicanas y españolas, desde la revisión de las políticas educativas
}

\author{
GUADALUPE PALMEROS Y ÁVILA* \\ JOAQUÍN GAIRÍN SALLÁN"
}

Universidad Juárez Autónoma de Tabasco - México

Recibido el 14-12-2015; primera evaluación el 26-01-2016; segunda evaluación el 12-04-2016; aceptado el 25-04-2016

\section{Resumen}

A nivel mundial, el tema de la inclusión en la educación superior ha cobrado mayor importancia en los últimos ańos y ha propiciado que se realicen acciones para la plena incorporación de los estudiantes En este trabajo se analizan los aspectos sustantivos y contextuales que están presentes en las universidades mexicanas y españolas en relación con la atención del estudiantado con discapacidad, considerando como eje el desarrollo histórico del proceso de inclusión educativa en ambos países e identificando las semejanzas, diferencias, necesidades y carencias para la atención de dichos estudiantes. De igual forma se analizan los distintos programas y acciones que se tienen para finalmente realizar algunas consideraciones para superar algunos factores que todavía persisten y limitan la eficacia en la atención a la diversidad así como la integración del colectivo de personas con discapacidad.

Palabras clave: acceso a la educación, discapacidad, educación superior, legislación, universidades.

\footnotetext{
Profesora investigadora de Tiempo Completo adscrita a la División Académica de Educación y Artes de la Universidad Juárez Autónoma de Tabasco impartiendo asignaturas en las licenciaturas en Ciencias de la Educación y Desarrollo Cultural. Pedagoga y maestra en Administración Educativa por parte de la Universidad Veracruzana y doctora en Calidad y Procesos de Innovación Educativa por parte de la Universidad Autónoma de Barcelona, España. Contacto: gpalmeros@hotmail.com

Profesor de EGB, Graduado social, psicólogo y pedagogo, actualmente es catedrático de Didáctica y Didáctica y Organización Escolar. Su experiencia docente e institucional es amplia, habiendo sido director de centro educativo, decano de facultad, comisionado del clúster en Educación y Formación, director de departamento universitario y del ICE de la Universidad Autónoma de Barcelona. Consultor internacional, está interviniendo en programas de reforma educativa en Espańa y Latinoamérica.
} 


\section{The care of people with disabilities in Mexican and Spanish universities}

\section{Abstract}

Worldwide, the subject of inclusion in higher education has become more important in recent years and has brought about actions for the full inclusion of students. In this work the substantive and contextual aspects that are present in Mexican and Spanish universities to the attention of students with disabilities are analysed, considering the historical development axis of the process of inclusive education in both countries and identifying similarities, differences, needs and deficiencies to the attention of those students. Likewise the different programs and actions that have to finally make some considerations to overcome some factors that still persist and limit the effectiveness in dealing with diversity and integration of the group of persons with disabilities will be analysed.

Keywords: Access to education, disability, higher education, legislation, universities.

\section{A atenção às pessoas com deficiência nas universidades mexicanas e espanholas, desde a revisão das políticas educativas}

\section{Resumo}

A nível mundial, o tema da inclusão no ensino superior adquiriu uma maior importância nos últimos anos e conduziu à realização de açôes no sentido da plena integração dos estudantes. Neste trabalho, analisam-se os aspetos concretos e contextuais que estão presentes nas universidades mexicanas e espanholas, relativamente aos estudantes com necessidades educativas especiais, considerando como eixo o desenvolvimento histórico do processo de inclusão educativa em ambos os países e identificam-se as semelhanças, diferenças, necessidades, e carências desses estudantes. De igual modo, analisam-se individualmente os dois programas e açóes para, finalmente, tecer algumas consideraçóes de modo a tornar possível ultrapassar alguns fatores que, apesar de tudo, persistem e limitam a eficácia da atenção à diversidade, tal como a integração das pessoas com deficiência.

Palavras-chave: acesso à educação, incapacidade, Educação superior, legislação, universidades 


\section{INTRODUCCIÓN}

Históricamente, las personas con discapacidad, ya sea por su limitación física, mental o sensorial o por barreras que la misma sociedad les impone han sido excluidas, rechazadas y marginadas al no permitirles el acceso a la educación, al empleo, a la protección social, a la salud, a la cultura, a los medios de transporte o a la información.

Los estigmas y barreras que sufren estas personas son enormes y las consecuencias aún más graves. En el caso de la educación se observa que, al no estar en igualdad de condiciones, presentan niveles más bajos en sus logros educativos. De hecho, son considerados un colectivo vulnerable que merece especial atención.

De acuerdo con las estimaciones de la población mundial en el año 2010 (OMS, 2011), más de mil millones de personas en el mundo viven con algún tipo de discapacidad; es decir, alrededor del 15\%, sin embargo, a pesar de que esta cifra es representativa, sufren diversos tipos de discriminación. Cortés, Garro, Cervini, Solano, Suárez y Castro (2009) mencionan dos razones que nos parecen importantes señalar para entender la situación de estas personas ya que les afecta de manera considerable: a) la estigmatización directa que viven por su condición; y b) por la falta de políticas y programas sociales adecuados para atender sus necesidades básicas.

En el caso de la educación, la incorporación de niños y jóvenes con discapacidad exige transformaciones importantes al sistema educativo en su totalidad, para lograr la equiparación de oportunidades. El éxito de estos sistemas inclusivos dependerá en gran medida del compromiso del país y de las mismas instituciones educativas para adoptar una legislación apropiada, adoptar planteamientos más centrados en el alumno, cambios en los planes de estudio, métodos y materiales de enseñanza, así como sistemas de evaluación.

Es por ello que, el presente documento tiene el objetivo de analizar la atención de los estudiantes universitarios con discapacidad en el contexto espańol y mexicano, dividiendo la exposición en apartados. El primero corresponde a la introducción, el segundo hace referencia a la discapacidad como preocupación, y su abordaje en la educación superior. En el tercero se mencionan las necesidades y carencias en la atención a los estudiantes universitarios con discapacidad. El cuarto y quinto incluyen la atención a los estudiantes universitarios con discapacidad en el contexto español y mexicano respectivamente, para después exponer en el sexto, los programas y acciones orientados a dichos estudiantes. Como último apartado se presentan algunas consideraciones finales, así como las referencias bibliográficas. 


\section{LA DISCAPACIDAD: UNA PREOCUPACIÓN EN LA EDUCACIÓN SUPERIOR}

Para la Organización Mundial de la Salud la discapacidad es:

Un término general que abarca las deficiencias, las limitaciones de la actividad y las restricciones de la participación. Las deficiencias son problemas que afectan a una estructura o función corporal, las limitaciones de la actividad son dificultades para ejecutar acciones o tareas, y las restricciones de la participación son problemas para participar en situaciones vitales. Por consiguiente, la discapacidad es un fenómeno complejo que refleja una interacción entre las características del organismo humano y las características de la sociedad en la que vive (OMS, 2011, p. 7).

Para una persona que presenta discapacidad, la falta o el inadecuado acceso físico al lugar de trabajo, escuelas, clínicas, transporte y edificios pueden representar un obstáculo, de ahí que, en los últimos años la discapacidad ha sido un motivo de preocupación por parte de organismos tanto nacionales como internacionales.

En el ámbito educativo, desde hace varias décadas se vienen realizando importantes esfuerzos a nivel mundial para garantizar el derecho a la educación sin ningún tipo de discriminación. Ya en el año de 1960, la Conferencia General de la Organización de las Naciones Unidas para la Educación, la Ciencia y la Cultura (Unesco) adopta la Convención relativa a la Lucha contra la Discriminación en la Esfera de la Enseñanza. Esta instancia condena la discriminación en el ámbito educativo y es la principal norma jurídica internacional e inicial que reconoce la existencia de acciones violatorias al principio de igualdad en los centros de enseñanza sin importar su grado.

A partir de la mencionada iniciativa, surgen estudios en los que se descubren las diversas formas en que se manifiesta la discriminación educativa. Uno inicial es el Informe Warnock (1978) de finales de los años setenta, que asume varios compromisos, entre ellos: a) que los jóvenes con necesidades especiales deben recibir el apoyo necesario para que puedan asistir a los cursos ordinarios de educación superior, b) que instituciones de educación superior ofrezcan cursos profesionales especiales a nivel operativo para los estudiantes con necesidades especiales y los cursos especiales de formación en competencia social y la independencia; y c) que cada autoridad local debe tener un centro de formación para la investigación, el desarrollo y en el servicio de educación especial para que los profesores puedan acudir en busca de ayuda para su desarrollo profesional. 
Estos compromisos han servido de referencia para la planificación y normativización de recursos educativos especiales en distintos países e instituciones de educación superior, han orientado aportaciones, nuevos planteamientos curriculares, así como nuevas formas de administración.

Lo anterior, da pautas para estructurar nuevas teorías sobre inclusión y profundizar en temas sobre la educación para la diversidad, promoviendo estudios que se enfocan desde la perspectiva de las personas con discapacidad. La universidad, como un espacio plural, debe desarrollar e impulsar desde sus funciones los proyectos vinculados a la problemática de la exclusión y diversidad para promover acciones encaminadas a fortalecerla como institución más inclusiva, de respeto, que reconozca a todos: De hecho, las universidades han de tratar de entender a la discapacidad como una condición de vida y, en consecuencia, el contexto universitario no debe limitar su desempeńo o impedir su acceso, permanencia y egreso en la educación superior.

Se espera, por tanto, que las universidades tomen conciencia del papel que les corresponde en la atención a las personas con discapacidad y, tal como se señala en el Manual para la integración de personas con discapacidad en las instituciones de educación superior,

la Universidad incluyente será más eficaz en la medida que utilice como puntos de partida las prácticas y conocimientos existentes; considere las diferencias como oportunidades, no como problemas; examine minuciosamente los obstáculos a la participación de los alumnos, haga un uso eficaz de los recursos disponibles para apoyar el aprendizaje; desarrolle un lenguaje común entre el profesorado, y cree unas condiciones que estimulen cierto grado de asunción de riesgos (ANUIES, 2002, pp. 19-20).

\section{NECESIDADES Y CARENCIAS EN LA ATENCIÓN A LOS ESTUDIANTES UNIVERSITARIOS CON DISCAPACIDAD}

Las necesidades y carencias en la atención a los estudiantes universitarios con discapacidad van a depender del tipo de discapacidad, las circunstancias personales y los factores ambientales, dado que los estudiantes tienen perfiles y demandas sociales diferentes. Verdugo y Campo (2005, p. 11) señalan que «no hay dos personas iguales ni dos discapacidades iguales, por lo que no habrá dos personas con discapacidad que necesiten lo mismo».

Por ejemplo, los estudiantes con discapacidad visual se caracterizan por tener dañada, en mayor o menor grado, la capacidad visual. En el contexto universitario, las personas ciegas o con baja visión pueden tener problemas para acudir a las clases, asistir a los exámenes, moverse con libertad por los 
centros — subir de planta, ir al baño, a la cafetería...-, etc. Es evidente que estas restricciones son todas ellas de carácter contextual y estarán íntimamente ligadas a las condiciones del entorno.

A su vez, los estudiantes con discapacidad auditiva «constituyen un grupo heterogéneo de personas que coinciden en tener alterada la función auditiva y que, en consecuencia, ven limitadas sus posibilidades de acceder al conjunto de informaciones sonoras del medio físico y social que les rodea» (Andreu y Navas, 2010, p. 94). Esta discapacidad no implica únicamente la incapacidad de oír o una disminución de la audición, sino que suele comportar una serie de consecuencias condicionadas por factores relacionados con la pérdida y por factores psicosociales, educativos y familiares de la persona. Las ayudas pueden ser de muy diversos tipos dependiendo del grado de pérdida auditiva y del estilo y habilidades comunicativas de cada persona: «desde un audífono a un colega que le toma apuntes, pasando por emisoras de frecuencia modulada, hasta un intérprete de signos o reservarle primeras filas si puede leer los labios del profesor» (Verdugo \& Campo, 2005, p. 12).

Con relación al estudiantado con movilidad reducida, los mencionados autores comentan que los principales obstáculos son las barreras arquitectónicas y el transporte. La falta de movimiento no tiene por qué repercutir de manera directa en los procesos cognitivos y de aprendizaje, aunque sí de manera indirecta por los tiempos de desplazamiento, de ejecución de las tareas, inaccesibilidad al manejo de documentación, cansancio físico, etc. Hay que ubicarlos en las aulas más accesibles, dar acceso a las pizarras y tarimas, proporcionar mobiliario adaptado, software y hardware específico, autorización para grabar las clases, que alguien tome apuntes, papel autocopiativo, entre otros.

Los ejemplos evidencian la particularidad de cada discapacidad y la necesidad de vincular la intervención con las necesidades individuales, considerando los recursos personales, materiales y didácticos necesarios para responder a ella. No cabe olvidar que este proceso debe estar centrado en la realidad del estudiante, en sus capacidades, no en sus limitaciones, y, sobre todo, en la posibilidad de modificarlas y desarrollarlas.

\section{LA ATENCIÓN A LOS ESTUDIANTES UNIVERSITARIOS CON DISCAPACIDAD EN EsPaña}

Aunque las personas con discapacidad están amparadas por los ordenamientos internacionales propios del sistema universal de derechos humanos, los países han impulsado la creación de instrumentos, leyes, normas, decretos y protocolos de actuación. En el caso de España se han generado diversos documentos 
que contienen planteamientos específicos con elación a los derechos de las personas con discapacidad y que a continuación se exponen.

La Estrategia Espańola sobre Discapacidad 2012-2020 del Ministerio de Sanidad, Servicios Sociales e Igualdad (2011) especifica que el número total de personas residentes que declaran tener alguna discapacidad asciende a $3847900-0,5 \%$ de la población-. De estas personas, 2,3 millones son mujeres lo que en términos relativos significa que, si de cada 100 hombres 7 dicen presentar una discapacidad, en las mujeres esta relación aumenta a 10 de cada 100. Los situados en el sistema universitario espańol serían 16279 , según datos del curso académico 2010-2011 (Rubiralta, 2011).

El marco legal que regula el principio de igualdad y no discriminación de las personas con discapacidad encuentra sus fundamentos en la Constitución Española de 1978 (Gobierno de España, 1978). Son varios los artículos en los que se menciona, de una u otra forma, el respeto a la diversidad y la consideración igualitaria de todos los españoles ante la ley. Así, se especifica de manera general en los artículos 9.2,10.1, 14, 7 y, de manera específica, el artículo 49 establece la defensa de los derechos de las personas con discapacidad, independientemente de su tipología: física, sensorial o psíquica.

Otra de las referencias básicas para entender el marco normativo español en materia de discapacidad es la Ley 13/1982, de 7 de abril, de Integración Social de los Minusválidos (LISMI) (Ministerio de la Presidencia, 1982) aún vigente, que constituye una referencia legislativa fundamental en el ordenamiento jurídico español de las personas con discapacidad, a pesar de fundamentarse en presupuestos de carácter rehabilitador. En su título VI, centrado en la temática de la rehabilitación, se dedica una sección específica a la educación.

La transición hacia el modelo social queda reflejada, en parte, en la Ley 51/2003, de Igualdad de Oportunidades, No Discriminación y Accesibilidad Universal de las personas con discapacidad (LIONDAU) (Ministerio de la Presidencia, 2003, s/p). Señala que «las desventajas que presenta una persona con discapacidad tienen su origen en sus dificultades personales, pero también y sobre todo en los obstáculos y condiciones limitativas que en la propia sociedad, concebida con arreglo al patrón de la persona media, se oponen a la plena participación de estos ciudadanos».

La aprobación de esta ley supone un cambio conceptual en la manera de entender el fenómeno de la discapacidad. Ya en el preámbulo se señala que es precisamente el diseńo y la concepción de la sociedad los que impiden la consecución de la igualdad real de las personas con discapacidad, implicando el desarrollo de un enfoque centrado en la lucha contra la discriminación y en la estrategia de accesibilidad universal. 
Otro avance que se ha producido es en el uso de la terminología empleada para hacer referencia a las personas con discapacidad, sustituyendo los términos peyorativos de minusválido, inválido, disminuido, entre otros, por persona con discapacidad.

Del Real Decreto 696/1995, de 28 de abril, de Ordenación de la Educación de los Alumnos con Necesidades Educativas Especiales (Ministerio de la Presidencia, 1995) es especialmente importante el artículo 18 relativo a los estudios universitarios. Se regulan las primeras medidas para propiciar la igualdad de oportunidades en el acceso a los estudios universitarios, instalaciones y enseñanzas. Además, se contempla la reserva de plazas para estudiantes con discapacidad.

También es un referente imprescindible para entender el marco jurídico, la Ley 6/2001, de 21 de diciembre, de Universidades, modificada por la Ley Orgánica 4/2007, 12 de abril (Ministerio de la Presidencia, 2007a). Uno de los aspectos más relevantes de esta norma, es la incorporación de medidas de acción positiva para las personas con discapacidad. En ella se estableció la importancia de impulsar políticas activas para garantizar la igualdad de oportunidades a las personas con discapacidad. También se estipula que las universidades debían dar respuesta a todas las demandas, así como cumplir, entre otros, con uno de los derechos fundamentales de los estudiantes: la igualdad de oportunidades y no discriminación por razones de sexo, raza, religión o discapacidad o cualquier otra condición o circunstancia personal o social en el acceso a la universidad, ingreso en los centros, permanencia en la universidad y ejercicio de los derechos académicos.

Asimismo, la Ley Orgánica de las Universidades establece entre las funciones de la Universidad, en su artículo 1, inciso c), la difusión, valorización y la transferencia del conocimiento al servicio de la cultura, de la calidad de vida y del desarrollo económico (Ministerio de la Presidencia, 2007a, p. 10). En la ley posterior, conocida como LOMLOU (Ley Orgánica 4/2007 del 12 de abril) también se otorgaron a las personas con discapacidad una mayor cobertura en el desarrollo de los procesos de aprendizaje universitarios.

El Real Decreto 1393/2007 de 29 de octubre -BOE, núm. 260, del 30 de octubre- (Ministerio de la Presidencia, 2007b) establece la Ordenación de las Enseñanzas Universitarias y dedica un apartado específico a las personas con discapacidad a la hora de organizar el acceso a las enseñanzas de grado. Su artículo 14.2 explicita que:

Las universidades dispondrán de sistemas accesibles de información y procedimientos de acogida y orientación de los estudiantes de nuevo 
ingreso para facilitar su incorporación a las enseñanzas universitarias correspondientes. Estos sistemas y procedimientos deberán incluir, en el caso de estudiantes con necesidades educativas específicas derivadas de discapacidad, los servicios de apoyo y asesoramiento adecuados, que evaluarán la necesidad de posibles adaptaciones curriculares (pp. 4-5).

Estos marcos normativos han impulsado acciones positivas en las universidades. De hecho, los estatutos de prácticamente la totalidad de las universidades públicas espańolas incluyen varios preceptos sobre el proceso de inclusión en contextos universitarios, específicamente para aquellos estudiantes con discapacidad. Algunos estatutos establecen la puesta en marcha de unidades de atención a los estudiantes con discapacidad, o de servicios específicos para este colectivo - asistencia social, inserción laboral, asesoramiento, orientación, tutorización, interpretación en lengua de signos, voluntariado-, entre otros. Además, existen estatutos y reglamentos universitarios que establecen medidas de adaptación curricular, sobre todo en lo que se refiere al tiempo y el formato de los exámenes, así como de apoyo en el ámbito de la docencia y la evaluación. Algunas universidades crearon incluso una comisión para llevarlas a cabo.

Destacamos especialmente la normativa más reciente de las señaladas, el Estatuto del Estudiante Universitario de 2011, precisamente por establecer tres cuestiones relevantes para nuestro foco de análisis: los departamentos en coordinación con el Servicio de Atención a la Discapacidad, determinan las adaptaciones metodológicas precisas; se concretan las tutorías específicas en función de las necesidades; y se promueven programas de tutoría permanente para que los estudiantes con discapacidad dispongan de un profesor tutor durante sus estudios (Rubiralta, 2011).

\section{LA ATENCIÓN A LOS ESTUDIANTES UNIVERSITARIOS CON DISCAPACIDAD EN MÉXICo}

En el caso de México, también se han generado una serie de instrumentos, leyes, normas, decretos y protocolos de actuación a nivel nacional y estatal que han propiciado la incorporación de personas con discapacidad.

Para el Instituto Nacional de Estadística y Geografía - Inegi (2010), una persona con discapacidad es aquella que tiene alguna limitación física o mental para realizar actividades en su casa, escuela o trabajo, como caminar, vestirse, bañarse, leer, escribir, escuchar, etcétera. Entre los tipos de discapacidad más conocidos señala la motriz, visual, mental, auditiva y de lenguaje. 
Menciona también que el tipo de discapacidad más frecuente es el de movilidad con el 58\%, — caminar o moverse-, le siguen las limitaciones para ver con un $8,2 \%$, escuchar con un $2,1 \%$, mental con $5 \%$, hablar o comunicarse con $8,3 \%$, atender el cuidado personal en un $5,5 \%$-vestirse, bañarse, comer-y, finalmente, poner atención o aprender un 4,4\%. Es decir, la dificultad para caminar o moverse y para ver representan el $85,5 \%$ de las discapacidades del país (Inegi, 2010).

De acuerdo con el Censo de Población y Vivienda 2010, habitan alrededor de 112 millones de personas. De ellas aproximadamente 5,7 millones - 5,1\% de la población total - son personas con discapacidad, siendo los relacionados con la movilidad y la vista los dos principales tipos.

Con relación al logro académico alcanzado, que para este grupo de población se relaciona con el acceso y la infraestructura, de la población con discapacidad mayor de 15 años, 27,9\% no tiene estudios, el 45,4\% terminó al menos un ańo de primaria, el 13,3\% uno de secundaria, el 7,3\% alguno de media superior y el 5,2\% uno en superior. El promedio de escolaridad es de $4,7 \%$. En cambio, las personas sin discapacidad presentan un perfil educativo más favorable, con un promedio de escolaridad de casi el doble - 8,9 años(Inegi, 2012).

De los jóvenes con discapacidad que tienen entre 15 y 29 años, solo el $15,5 \%$ asiste a la escuela, casi $10 \%$ de la población total del país que tiene 15 años y más no sabe leer y escribir; en la población con discapacidad este dato representa un 32,9\%. Por otra parte tenemos que, de cada 100 personas de 15 años y más con discapacidad, 36 no cursan ningún grado escolar, 46 tienen educación básica — primaria y secundaria incompleta—, 7 tienen educación básica completa, 5 han cursado algún grado de educación media superior y 4 cuentan con educación superior (Inegi, 2012).

El derecho a la educación en México, se encuentra consagrado en el Artículo Tercero Constitucional, el cual señala que «todo individuo tiene derecho a recibir educación» (Gobierno de México, 2013, s/p). A su vez, en el artículo 41 de la Ley General de Educación (Diario Oficial de la Federación, 2015) se menciona que «la educación especial está destinada a personas con discapacidad, transitoria o definitiva así como aquellas con aptitudes sobresalientes. Atenderá a los educandos de manera adecuada a sus propias condiciones, con equidad social incluyente y con perspectiva de género» (p. 8).

En este mismo sentido, la Ley Federal para Prevenir y Eliminar la Discriminación (Diario Oficial de la Federación, 2014) establece como objetivo "prevenir y eliminar todas las formas de discriminación que se ejerzan 
contra cualquier persona en los términos del artículo 1 de la Constitución Política de los Estados Unidos Mexicanos, así como promover la igualdad de oportunidades y de trato» (p. 1).

Por otro lado, entre los documentos normativos que se refieren a la atención a las personas con discapacidad, se encuentra la Norma Oficial Mexicana NOM-173-SSA1-1998 para la Atención Integral a Personas con Discapacidad; la Norma Oficial Mexicana NOM-233-SSA1-2003, que establece los requisitos arquitectónicos para facilitar el acceso, tránsito, uso y permanencia de las Personas con Discapacidad; y el Manual de Accesibilidad del Programa Nacional de Fortalecimiento de la Educación Especial y de la Integración Educativa.

De igual manera, existen organizaciones gubernamentales encargadas de promover, atender, reconocer y apoyar acciones en materia de discapacidad en México, siendo estas: El Consejo Nacional de Personas con Discapacidad (Conadis), cuyo objetivo es contribuir al establecimiento de una política de Estado en la materia, así como promover, apoyar, fomentar, vigilar y evaluar las acciones, estrategias y programas derivados de la Ley General de Personas con Discapacidad, en su artículo 29.

Por otra parte, la Secretaría de Educación Pública (SEP) desde hace décadas ha enfrentado el enorme reto de ofrecer mayores oportunidades de acceso a los niños y jóvenes que presentan necesidades educativas especiales (NEE), con o sin discapacidad, a una educación con equidad, que los atienda de manera adecuada a sus propias condiciones.

\section{Programas y acciones}

En los últimos años, se ha observado un incremento en el ingreso de estudiantes con discapacidad en las universidades, que ha generado que se realicen programas y acciones encaminados a la atención de estos estudiantes con el objeto de ofrecer igualdad de oportunidades tanto en el acceso como en la permanencia y egreso. Un programa de atención a personas con discapacidad es una estrategia que cada institución, de acuerdo con las necesidades específicas y de atención a sus estudiantes, diseña para promover la prevención a través del equipamiento y capacitación de instancias que atienden a personas con discapacidad. De manera general se destacan y se pueden identificar en España y México las siguientes actuaciones ANUIES (2002), SEP (2015, 2013a 2013b), Freixa, Venceslao y Pol (2011) y Rubiralta (2011): 
1. Programas de apoyo académico

a) Tutorización y/o seguimiento. Programas que tienen como propósito orientar y apoyar a los estudiantes con discapacidad durante su proceso de formación universitaria a partir del conocimiento de sus problemas y necesidades académicas, así como de sus inquietudes y aspiraciones profesionales.

b) Cursos, formaciones y jornadas. Orientadas al ámbito de la discapacidad, se imparten o realizan proporcionando información y formación adecuadas y basadas en los principios de los derechos humanos.

c) Diseño de los planes de estudio, que consideran la variable de la discapacidad. Aquí se deben considerar cambios en el acceso, metodología y contenidos curriculares.

d) Materiales de apoyo, como puede ser el Plan de acción tutorial para estudiantes universitarios con discapacidad: Guía para su concreción, que proporciona orientaciones para gestores y profesorado universitario implicado en la temática (Galán, Gairín, Fernández, Sanahuja, Muñoz y Oliver, 2012).

e) Adaptaciones curriculares para estudiantes con discapacidad. Dos estudiantes que presenten la misma discapacidad pueden requerir diferentes ajustes curriculares, evitando generalizar las situaciones.

2. Programas de apoyo económico

a) A través del otorgamiento de becas se pretende impulsar el acceso, el progreso y la movilidad en el proceso de formación académica universitaria hasta la finalización de los estudios. Estas becas consideran la exención de pago de matrícula, descuentos en las tasas de matriculación o asignación de una determinada cantidad de dinero, ya sea mensual o semestral.

3. Programas de apoyo estructurales

a) Accesibilidad de las instalaciones en entornos físicos que implica la posibilidad de una persona de ingresar, transitar y permanecer en un lugar, de manera segura, confortable y autónoma.

b) Creación de servicios de apoyo a los estudiantes universitarios con discapacidad.

4. Otros programas de apoyo

a) Desarrollo de planes de acción tutorial por facultades y carreras 
b) Accesibilidad y diseño para todos en entornos virtuales. Hace referencia a la posibilidad existente para adaptar los contenidos educativos de las TIC a diversos tipos de discapacidad.

c) Programas específicos de ocio accesible y deporte adaptado. Se trata de asegurar que las personas con discapacidad tengan la oportunidad de organizar y desarrollar actividades deportivas y recreativas específicas para ellas.

d) Acciones de apoyo a la empleabilidad y la inclusión laboral. Conjunto de acciones diseñadas para conseguir el acceso y el mantenimiento en el empleo de las personas.

e) Intérprete de Lenguas de Signos Española (LSE). El papel de estas personas es hacer un puente de comunicación entre la comunidad sorda y la comunidad oyente. Para garantizar el derecho al acceso a la información, se debe de recurrir a estos profesionales formados para tal fin.

f) Asistencia personal. Es necesaria para facilitar su existencia y su inclusión en la comunidad académica al proporcionarle la ayuda que necesita la persona con discapacidad, pero, al mismo tiempo, otorgándole un control entero del proceso del usuario, para garantizar que se adapta a sus necesidades y directrices necesarias para su desempeño escolar.

Sirva también como referencia del nivel de desarrollo alcanzado, la síntesis de la realidad española realizada en un reciente estudio por Galán, Gairín, Fernández, Sanahuja y Oliver (2013).

- Mayoritariamente las actuaciones se focalizan en la permanencia de los estudiantes con discapacidad en la universidad. En segundo lugar, las actuaciones relacionadas con la acogida y el egreso de los estudiantes con discapacidad y las actuaciones del ámbito de la promoción son las que están menos desarrolladas por los servicios, unidades u oficinas de atención al estudiante con discapacidad en la universidad.

- Ninguno de los Servicios, Unidades u Oficinas analizados dispone de un Plan de Acción Tutorial de carácter global (tampoco los hay centrados en un momento de transición) y dirigido al colectivo de estudiantes con discapacidad; las actuaciones de orientación y tutoría que realizan responden así a momentos y situaciones más concretas y específicos.

- Las actuaciones para la promoción son, fundamentalmente, acciones de carácter informativo que procuran dar a conocer los servicios, 
unidades $\mathrm{u}$ oficinas de atención al estudiante con discapacidad que tienen las universidades.

- Las actuaciones vinculadas a la acogida se centran en facilitar información de interés para el colectivo de estudiantes con discapacidad. La formación y el asesoramiento al profesorado, las familias y el personal de administración y servicios se va instalando poco a poco; a veces, incluso, se elaboran guías de orientación y apoyo al estudio para estos tres colectivos y para los estudiantes con discapacidad.

- Las actuaciones para la permanencia se centran en el seguimiento y la orientación académica al estudiante, seguidas de la personal. Una importante parte de los esfuerzos dedicados por los servicios, unidades u oficinas, se sitúa en dar soporte a los estudiantes para el desarrollo de habilidades sociales y para el estudio apuntes, técnicas de estudios, entre otros, contando con el apoyo y colaboración entre iguales a través de redes de voluntariado.

- Las actuaciones para el egreso se centran en la orientación laboral y poco en la orientación académica para que el estudiante con discapacidad pueda continuar estudiando. Todavía son pocas las universidades que establecen convenios de colaboración con centros de trabajo para la inserción laboral de los estudiantes titulados con discapacidad.

- Otras actuaciones frecuentes que se desarrollan tienen que ver con la mejora de la accesibilidad integral supresión de barreras arquitectónicas, adecuación de espacios, eliminación de barreras comunicativas, [...]; actividades sociales, deportivas y de ocio; servicios adaptados bibliotecas, alojamientos, [...]; y la sensibilización de la comunidad por un sistema universitario más integrador e inclusivo.

Las investigaciones del Grupo TRALS (Transiciones Académicas y Laborales) de la Universidad de Barcelona (Freixa, Venceslao \& Pol, 2011), también subrayan la importancia de que los Servicios Universitarios de Atención a Estudiantes con Discapacidad puedan aproximarnos a la consecución de la plena inclusión a través de planes individualizados para la transición de las personas con discapacidad, que deben empezarse dos años antes de terminar la enseñanza secundaria; unos servicios universitarios que no solo informan y acogen, sino que también mejoran las dinámicas curriculares; el seguimiento personalizado del estudiantado a lo largo de todo el proceso; un trabajo cooperativo, desde la corresponsabilidad de las familias y del contexto social; y la profesionalización del personal encargado de la orientación, la instrucción y la evaluación. 
Mantener lo conseguido o mejorarlo no es fácil, pero si factible como un reto que progresivamente puede ser superable. La opción es importante y, por lo mismo, debe de ser abierta a todos los estudiantes y a todas las posibilidades.

Supone, además, considerar y hablar de la responsabilidad del profesor — que debe ser receptivo - y del alumno — que debe de ser proactivo, dando a conocer sus necesidades, solicitando servicios, brindando información sobre su situación o reclamando todo lo que precise-; de la manera cómo lo comunica y cómo lo puede comunicar el estudiante discapacitado; de la manera cómo el estudiante percibe y siente la relación de ayuda, que puede exigir, a veces, la mediación; y de trabajar desde la normalidad.

Progresivamente, se trata de superar la universidad segregadora y avanzar en la línea de la integración y en el reto de la inclusión. La universidad inclusiva piensa en todas y cada una de las personas que con ella se relacionan y toma medidas para que esas relaciones no queden afectadas por las características de las personas.

\section{Algunas consideraciones finales}

En el aspecto normativo, tanto en España como en México, la revisión documental remarca que hay un desarrollo importante a nivel de políticas públicas, que recogen compromisos internacionales y una apuesta por la atención a la discapacidad en la universidad. Sin embargo, factores diversos - limitación de recursos, planes de integración no totalmente desarrollados, profesorado no acostumbrado a trabajar la diversidad, entre otros - limitan la realidad de una escuela inclusiva. Los estudios de Cortes, Garro, Cervini, Solano, Suárez y Castro (2009), Verdugo y Campo (2005) y más recientemente el Proyecto ACCEDES (Acceso, permanencia y egreso de colectivos vulnerables en Latinoamérica), remarcan que no todas las universidades tienen servicios, unidades y programas de apoyo a los estudiantes con discapacidad y que sus actuaciones quedan limitadas a una parte de la población objetivo.

En el aspecto académico, las tutorías, la implementación de cursos, formación de profesionales, la realización de jornadas, el diseño del plan de estudios, así como las adaptaciones curriculares, han sido implementadas poco a poco en las universidades. Si bien existen avances en ello y los logros han sido significativos en cuanto a la discapacidad motriz y visual, aún faltan mucho por hacer respecto a otras discapacidades.

La inclusión pretendida representa, de alguna manera y siguiendo a Sebba y Ainscow (1996), un proceso por el que la institución educativa trata de dar respuesta a todos los individuos como estudiantes y reconstruye el currículum 
para llegar a todos los estudiantes individualmente. Para estos mismos autores, en el desarrollo de unas prácticas más inclusivas es necesario considerar los procesos conectados de inclusión y exclusión; y que el apoyo educativo de los profesionales de la educación sobre algunos grupos de estudiantes con necesidades es requerido para responder a la diversidad en determinadas fases y momentos.

De lo que se trata es de eliminar la exclusión por diferencias de capacidad, raza, clase social, etnia, género, entre otros, para aprovechar las potencialidades de todas las personas y sus posibilidades de crecimiento y desarrollo para aprender y convivir de forma conjunta, solidaria y cooperativa, desde la aceptación de la diferencia y el reconocimiento de las identidades individuales y colectivas. También es importante pasar de las actuaciones generales sobre la diversidad a las actuaciones específicas y focalizadas en los colectivos que precisan atención particularizada (como es el caso, de algunos grupos de discapacitados).

En palabras de Arnáiz, cabe esperar que la educación inclusiva «contribuya a desarrollar la ansiada cohesión social convirtiéndose en un elemento indispensable para que la sociedad pueda progresar hacia los ideales de paz, libertad y justicia social; un servicio público que beneficie el desarrollo humano en condiciones de igualdad, no constituyéndose en un factor adicional de exclusión» (2012, p. 41).

Por eso, conviene superar algunos factores que todavía persisten y que limitan la eficacia en la atención a la diversidad y la integración del colectivo de personas con discapacidad. De acuerdo con Gurdián (2002),

Una imagen un tanto distorsionada de la persona con discapacidad en la práctica y por la desinformación, los prejuicios y la forma por cómo se concibe la normalidad; una sociedad que no ofrece del todo igualdad de oportunidades, ni asume la diversidad; y el insuficiente reconocimiento de las personas con discapacidad como sujetos con capacidades y limitaciones como todos los seres humanos (p. 127).

De acuerdo con Loyo y Calvo (2009) y Sinisi (2010), parece importante remarcar también la necesidad de volver la mirada hacia las actuaciones institucionales propias de la organización y de la clase, e implicar a todos los actores para analizar qué factores, prácticas y creencias, estarían operando en las trayectorias educativas que continúan sin completarse. Así, la efectividad de las actuaciones centradas en los discapacitados, son más efectivas si cuentan con el trabajo conjunto y colaborativo de los servicios de apoyo, de los asesores de la discapacidad de que se trate, del profesorado y del estudiantado afectado. 
También para Stainback y Stainback (1999) serían las mismas instituciones las que deben de asumir la responsabilidad de apoyar a todos los estudiantes en el logro de sus objetivos académicos, promoviendo programas y acciones en su interior y que ofrezcan la oportunidad de ingresar, permanecer y egresar de la Educación Superior en igualdad de condiciones (Lissi, Zuzulich, Salinas, Achiardi, Hojas \& Pedrals, 2009).

Las estrategias institucionales efectivas se relacionan así con las lógicas y dinámicas internas y con el contexto social, cultural, político y económico en que se encuentran estas instituciones (Rezaval, 2008). Así, es posible reconocer que algunos de los aspectos que afectan al tratamiento de las personas con discapacidad están siendo trabajados y recogidos por diferentes experiencias e iniciativas en las universidades, que promueven experiencias inclusivas en su interior y en vinculación con la sociedad y el contexto.

Dicha transformación no solo se relaciona con los sistemas de acceso y de selección sino también con aspectos, en muchas ocasiones constituidos como barreras, tales como la disponibilidad de la oferta académica, planes de estudios y métodos de evaluación poco flexibles, pero también cuestiones de financiamiento y presupuesto (Blanco, 2009). Asimismo, tal y como plantean Sebastián y Scharager (2007), la diversidad también debe ser analizada a nivel de los académicos y a nivel institucional — del profesorado y de la institución misma-, lo que complejiza y, a la vez, hace más integral el enfoque sobre la inclusión en la educación superior.

De todas formas, el proceso para ser efectivo debería de ser sistemático y continuo, sin embargo, la falta de planes tutoriales en la mayoría de las universidades (Galán et. al., 2012) y la baja incorporación a los mismos de actuaciones específicos para colectivos discapacitados u otros, actúan en sentido contrario.

\section{REFERENCIAS BIBLIOGRÁFICAS}

Andreu, A.B. y Navas, N. (2010). Estudiantes con discapacidad en la Educación Superior: Necesidades y orientaciones para la intervención. En Víctor Rodríguez (Coord.), Atención a los estudiantes con discapacidad en la universidad (pp. 91-123). Madrid: UNED.

ANUIES (2002). Manual para la Integración de Personas con Discapacidad en las Instituciones de Educación Superior. México D.F.: ANUIES.

Arnáiz Sánchez, Pilar (2012). Escuelas eficaces e inclusivas: cómo favorecer su desarrollo. Educatio Siglo XXI, 30(1), 25-44. Disponible en: http://revistas. um.es/educatio/article/view/149121 
Blanco R. (2009). Acceso e inclusión en Educación Superior: de lo posible a lo necesario. En Francisco J. Gil y Jaumet Bachs (Eds.), Una experiencia exitosa por una educación superior más inclusiva (pp. 48-57). Santiago de Chile: USACH - UNESCO - Fundación Equitas.

Cortés, M., Garro, N., Cervini, H., Solano, C., Suárez, M. y Castro, L. (2009). Programa de Atención a Personas con Discapacidad. Cuadernos del Consejo de Desarrollo Social 15. Gobierno del Estado de Nuevo León, Recuperado de http://www.nl.gob.mx/pics/pages/cuadernos_cds_base/libro15.pdf

Diario Oficial de la Federación (2014). Ley federal para prevenir y eliminar la discriminación. México. Secretaría de Gobernación. Diario Oficial de la Federación, 20 de marzo de 2014.

Diario Oficial de la Federación (2015) Ley General de Educación. México, Secretaría de Educación Pública. Diario Oficial de la Federación, 17 de diciembre de 2015.

Freixa, M., Venceslao, M. \& Pol, C. (2011). Servicios universitarios de atención a estudiantes con discapacidad: hacia una Universidad más inclusiva. En $X V$ Congreso Nacional y I Internacional de Modelos de Investigación Educativa, del 21 al 23 de septiembre de 2011, Madrid, UNED - Asociación Interuniversitaria de Investigación Pedagógica AIDIPE.

Galán A., Gairín J., Fernández, M., Sanahuja J.M., Muñoz, J.L. \& Oliver, J. (2012). El plan de acción tutorial para estudiantes universitarios con discapacidad (PATdis) Guia de Recomendaciones. Madrid: Ministerio de Educación, Cultura y de Deportes, Recuperado de http://accelera.uab.cat/ documents_edo/pdf/Guia_Plan_Accion_Tutorial.pdf

Galán, A., Gairín, J., Fernández, M., Sanahuja J.M. \& Oliver, J. (2013). Plan de acción tutorial para estudiantes universitarios con discapacidad. Madrid: Ministerio de Educación, Memoria del Proyecto AZ510131.

Gobierno de España (1978). Constitución Española. BOE, 311, 29 de diciembre de 1978.

Gobierno de México (2013). Constitución Política de los Estados Unidos Mexicanos. Diario Oficial de la Federación, 5 de febrero de 1917. Última reforma publicada DOF 19-07-2013, Recuperado de http://www.diputados.gob.mx/LeyesBiblio/pdf/1.pdf

Gurdián, A. (2002). Rompiendo prejuicios y provocando rupturas. En R. Jiménez (Ed.), Las personas con discapacidad en la educación superior (pp. 127-139). San José: Fundación Justicia y Género.

Instituto Nacional de Estadística y Geografía (2010). Censo de Población y Vivienda 2010. Recuperado de: http://www.inegi.org.mx/lib/olap/consulta/general_ver4/MDXQueryDatos.asp? 
Instituto Nacional de Estadística y Geografía (2012). Estadísticas a propósito del dia internacional de las personas con discapacidad. Aguascalientes, Ags, Recuperado de www.inegi.org.mx/inegi/contenidos/espanol/.../discapacidad14.doc

Lissi, M.R., Zuzulich, S., Salinas, M., Achiardi, C., Hojas. A.M. \& Pedrals, N. (2009). Discapacidad en contextos universitarios: experiencia del PIANE UC en la Pontificia Universidad Católica de Chile. Calidad en la Educación, 30, 305-324.

Loyo, A. \& Calvo, B. (2009). Centros de Transformación Educativa. México D.F. Madrid: Fundación Iberoamericana para la Educación, la Ciencia y la Cultura.

Ministerio de la Presidencia (1982). Ley de integración social de los minusválidos (LISMI), Ley 13/1982, BOE, 103, 30 de abril de 1982. España. Gobierno de España.

Ministerio de la Presidencia (1995). Real Decreto 696/1995 de 28 de abril, de Ordenación de la Educación de los Alumnos con Necesidades Educativas Especiales. BOE, 131, 2 de junio de 1995. España. Gobierno de España.

Ministerio de la Presidencia (2003). Ley de igualdad de oportunidades, no discriminación y accesibilidad universal de las personas con discapacidad (LIONDAU). BOE, 289, 3 de diciembre 2003. España. Gobierno de España.

Ministerio de la Presidencia (2007a). Ley Orgánica 4/2007, de 12 de abril, por la que se modifica la Ley Orgánica 6/2001, de 21 de diciembre, de Universidades. BOE, 89, 13 de abril de 2007. España. Gobierno de España.

Ministerio de la Presidencia (2007b) Real Decreto 1393/2007 de 29 de octubre, por el que se establece la Ordenación de las Enseñanzas Universitarias. BOE, 260, 30 de octubre de 2007. España. Gobierno de España.

Ministerio de Sanidad, Servicios Sociales e Igualdad (2011). Estrategia Española sobre Discapacidad 2012-2020. Madrid, Real Patronato sobre Discapacidad. España. Gobierno de España.

Organización Mundial de la Salud (2011). Informe Mundial sobre la Discapacidad. Suiza: Ediciones de la OMS. Recuperado de http://www.who.int/ disabilities/world_report/2011/summary_es.pdf

Rezaval, J. (2008). Politicas de inclusión social a la educación superior en Argentina, Chile y Perú. Buenos Aires: Facultad Latinoamericana de Ciencias Sociales - FLACSO, Sede Académica Argentina.

Rubiralta, M. (Coord.) (2011). Las politicas sobre la discapacidad en el sistema universitario español. Gobierno de España: Ministerio de Educación Secretaría General de Universidades. 
Sebastian, C. \& Scharager, J. (2007). Diversidad y educación superior: algunas reflexiones iniciales. Calidad en la Educación, 26, 19-36.

Sebba, J. \& Ainscow, M. (1996), International developments in inclusive schooling: mapping the issues. Cambridge Journal of Education, 26(1), 5-18.

Secretaría de Educación Pública (2013a). Programa Sectorial de Educación 20132018. México: SEP.

Secretaría de Educación Pública (2013b). Necesidades educativas especiales, Recuperado de http://www.sincree.sep.gob.mx/en/controlescolar/ Necesidades_Educativas_Especiales

Secretaría de Educación Pública (2015). Necesidades Educativas Especiales. Recuperado de http://www.sincree.sep.gob.mx/en/controlescolar/ Necesidades_Educativas_Especiales

Sinisi, L. (2010). Integración o Inclusión escolar: ¿̨un cambio de paradigma? Boletín de Antropología y Educación, 1, 11- 14.

Stainback, S. \& Stainback, W. (1999). Aulas inclusivas. Madrid: Narcea.

Verdugo, M.A. \& Campo, M. (2005). Análisis de servicios y programas universitarios de apoyo a estudiantes con discapacidad en las universidades Españolas. Informe final. Instituto Universitario de Integración en la Comunidad. Recuperado de http://sid.usal.es/idocs/F8/FDO17238/analisis_servicios.pdf.

Warnock Report (1978). Special Educational Needs Report of the Committee of Enquiry into the Education of Handicapped Children and Young People. London: Her Majesty's Stationery Office 1978. Recuperado de http:// www.educationengland.org.uk/documents/warnock/ 